\title{
Introduction to the Function of Bisokotuwa in Ancient Vewa
}

\section{Kapila Peiris and Sanjeewa Wijesinghe}

Abstract: This brief paper deals with an introduction of analyzing the function of the Bisokotuwa, a structure found in ancient vewas (water reservoir) in Sri Lanka in the domain of modern Newtonian fluid mechanics and development of an analysis to investigate the possibility of using the Bisokotuwa as a flow rate meter in vewas.

\section{Introduction}

Sri Lanka has one of the most glorious ancient irrigation systems in the world. Though it is named as an irrigation system, today most renowned authorities in this field called this as a sustainable soil water ecosystem ${ }^{(3)}$ This soil water ecosystem was mainly spread in an area of the country, which is known today as the dryzone. The system mainly consists of small and big vewas and interconnected canals. The main purpose of this system was to maintain a sustainable water level (mainly in soil) in the environment and thereby to enhance the lives of not only human but animals, birds etc.. in the environment. Other than in the sections where some structures have been destructed by foreign invasions and under modern "Development" schemes this water-soil-ecosystem has been functioning satisfactory for more than thousand years. What were the theories, concepts and knowledge that our ancestral engineers possessed to build such systems? What were their fluid mechanics? Or rather did they have any theories? As most of our scholars say today were they just manipulating with empherical results? What is the demarcation boundary between empherical results and theories? How did our ancient engineers determine the size of a "Bisokotuwa" in relation to a particular vewa? In the investigation of the "Bisokotuwa" (or any other part in this soil-water-ecosystem) we must first answer the above-mentioned questions in a background based on our (Sinhala) culture.

Also it should be mentioned here that in ancient times it is hardly reasonable to think that there were separate subjects such as science technology, arts, development economics, religion etc. All these subjects which we see separately today would have been in a single wholistic system which was governed, guided by a world view or a life philosophy. Therefore, the most important aspect in analyzing or doing research on ancient "technology" is to find out this world view, economic development model and life philosophy that would have given rise to these "technologies"

Two of the most important components in a vewa were its sluice and sluice gate. These components must control the outflow of water from vewa, without damaging the bund (dam) and related cannels from pressure and velocity of water.

The out going water from small village level tanks were controlled by using temporary cuts in the earthen banks. In medium size tanks this was done by using a technique called Keta Sorrowva (Figure 1) The flow rate of the out going water from the vcwa was controlled by a number of "Keta" kept one on top of the other ${ }^{(1)}$ By this method the head of water entering the sluice was controlled. In much bigger tanks (Vewa) with depths of about 30-40 ft. like Nuwara vewa, Tissa vewa, Kala vewa at Anuradhapura, Urisita vewa at Sooriyavawa etc..controlling the out going water by the above method is not possible. This is mainly due to the high head of the vewa. In such situations our ancestral engineers have invented a marvelous structure to fulfill the above purpose. This is known as the "Bisokotuwa" (figure 2). This structure attached to the sluice could be very efficiently used to control the flow rate of water from vewa without exerting high pressure and velocities on the bund (dam) as well as acting as a surge tank in opening and closing the gates of the vewa.

Eng. Kapila Peiris, BSc Eng., C.Eng., MIE(SriLanka), Presently, Mechanical Engineer, NERD Centre of Sri Lankn

Eng. Sanjeewa Wijesinghe,BSc Eng., C.Eno., MIE(Sri Lanka), Presently, Project Manager, Central Engineering Consultancy Burenu 

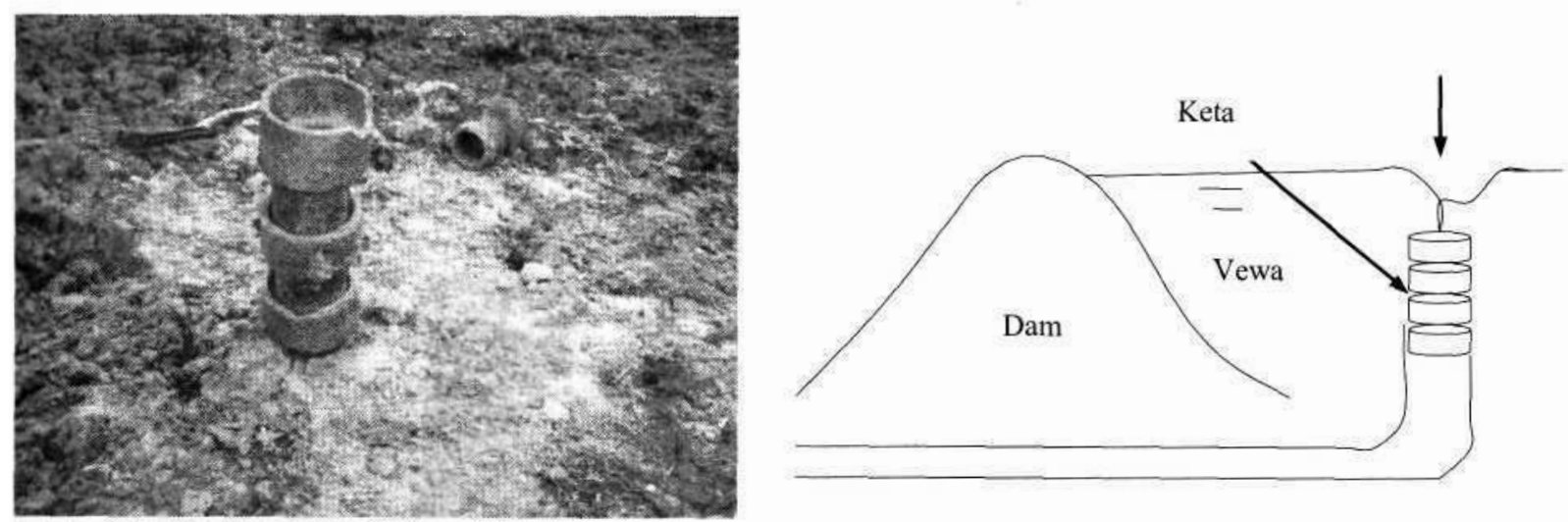

Figure 1: Keta sorrowva

In most cases this is an enclosure 8-12 ft long and 8-10 $\mathrm{ft}$ wide, built across the culvert in the up stream side of the dam. It has thick walls of brick work laid in mortar, around which there is an excellent water tight backing of tempered claya "Puddle". At most works the brick is faced or lined inside the well with admirably cut thin slabs of stone laid horizontally and invariably on their edges, which fit closely together ${ }^{(4)}$. The purpose of all these construction methodologies would be to prevent water leakage to the bund from Bisokotuwa.

\section{Analyzing the Function of Bisokotuwa}

As shown in figure 2 Bisokotuwa consists of a rectangular tank connected to the sluice, in almost all the cases the longer side of the rectangle is kept parallel to the bund. Though any evidance regarding the gates of the Bisokotuwa is not found up to now, the speculated gates which would have been made out of wood and controlled by a system of levers, shown in figure 2 are mostly accepted by present day scholars. With these speculated gates the function of Bisokotuwa could be understood as follows.

Lifting arrangement

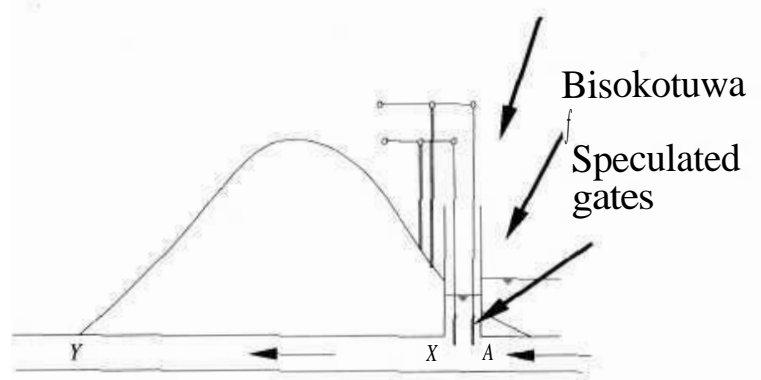

Figure 2: Schematic diagram of Bisokotuwa

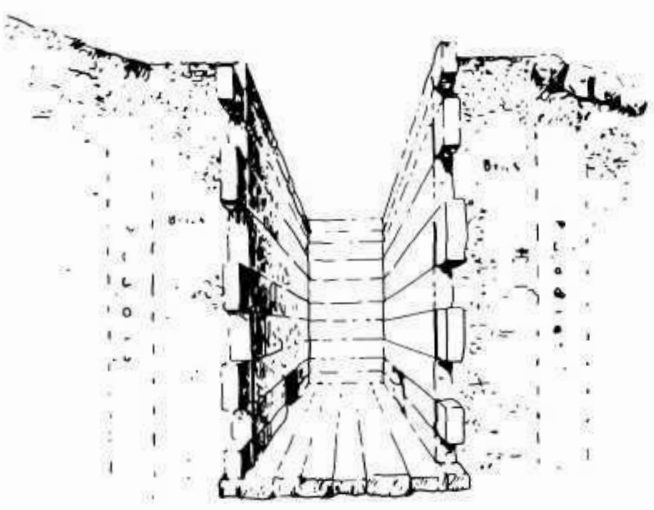

Figure. 3: Inside view of Bisokotuwa [4]

When it is required to release water from vewa gate "A" is opened gradually, while B as kept open with a particular opening (or gate B would have not existed). Then water level in the Bisokotuwa will come to a height less than that of the water level in vewa. Then water will flow through sluice to the outside canal driven by the head of the Bisokotuwa, without subjecting the sluice to high pressure as well as velocities created directly from the head of vewa. When stopping the water released from the vewa, gate "A" is closed then the water inside the Bisokotuwa will flow through sluice XY (Figure 2) without creating any vacuum condition. In this manner "Bisokotuwa" acts as a surge tank but this is not a mere surge tank. If this was only a surge tank such large cross sections as mentioned are not needed.

According to figure (4) when water entering Bisokotuwa water will be subjected to severe velocity gradients. [This may be probably the reason for laying the Bisokotuwa in such a way that its longer side is parallel to the bund i.e perpendicular to the sluice (the direction for water flow)]. Due to these high velocity gradients shear stresses will be formed within water and these shear stresses will reduce the 
pressure of water. In other words the shear forces that formed are mainly responsible for the deceleration of water entering the Bisokotuwa. These shear forces are responsible for the energy dissipation in the Bisokotuwa.

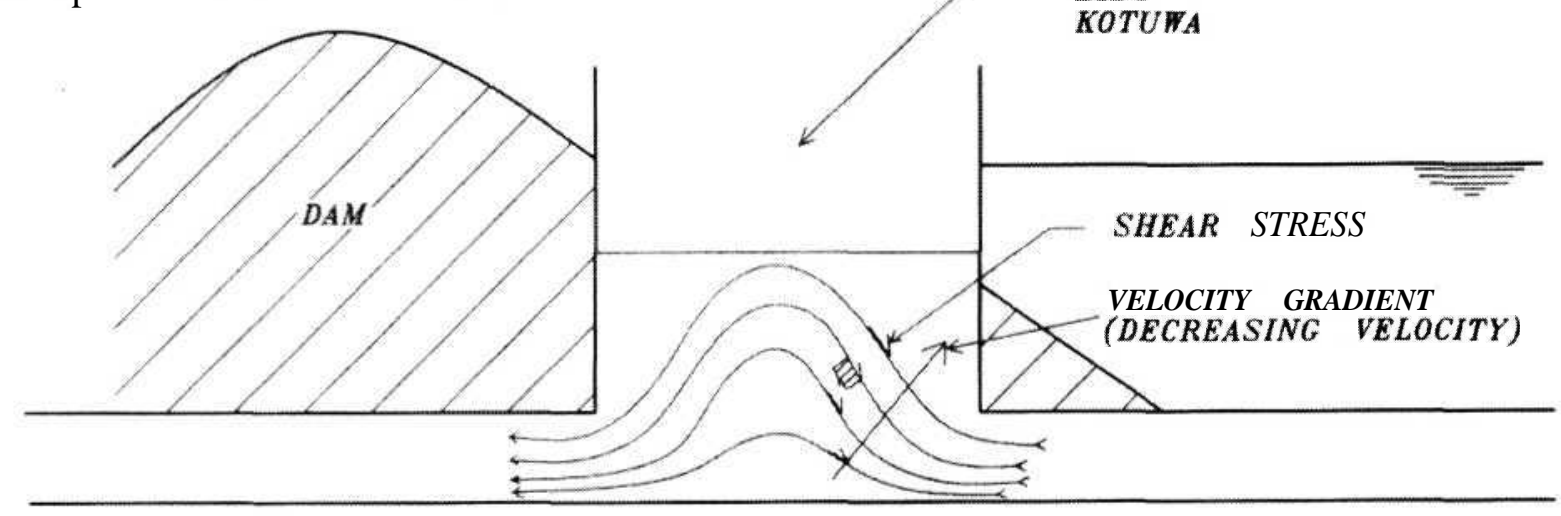

Figure 4: Due to high velocity gradient, the velocity of inlet water is reduced by shear stress instead of increasing of pressure.

When water is going out from the vewa, first water enters the Bisokotuwa. In the Bisokotuwa the head of water entering it is decreased by subjecting it to higher shear stress. Bisokotuwa is a non-destructive energy dissipation tank. For this to take place properly the volume of water inside the Bisokotuwa is important. The large cross sections of Bisokotuwa stated above will fulfill this requirement. If the volume of Bisokotuwa is too small the formation of above mentioned high velocity gradients shear stressess and pressure drop will not take place. Also when the width of the Bisokotuwa (side parararel to the bund) is large, diversion of stream lines in that direction will be more and shear forces will take place resulting in high energy losses. In some vewas for example Urusita vewa at Sooriyavewa, water enters Bisokotuwa by one conduit (sluice) at the bottom centre and goes out by two conduits beginning from the bottom of wall (Figure 5). With this arrangement the effect of momentum of inlet water to outlet water will be minimized i.e. out flow will be much calmer. By looking at the sluices within the dams and at sketches by Parker of water flow within these sluices it could be inferred that the water flow within these sluices would have been mainly free gravity flow. Bisokotuwa is also used to divert water flows. One such example is seen at a outlet from Parakkramasamudra (Figure 6). In this case water enters the Bisokotuwa on one side and leaves it at the perpendicular side. The forces required to divert the flow of water is obtained from water itself (inside the Bisokotuwa)
Therefore no effects such as erosion of conduits will take place.

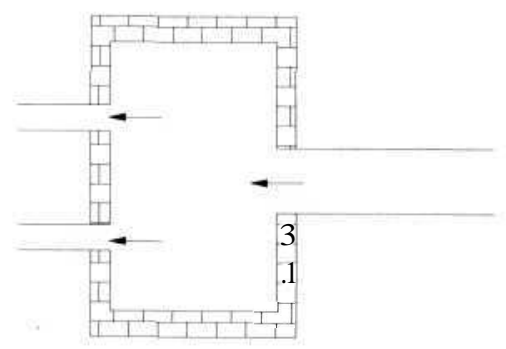

Figure. 5: Bisokotuwa at Urusita weva (Plan)
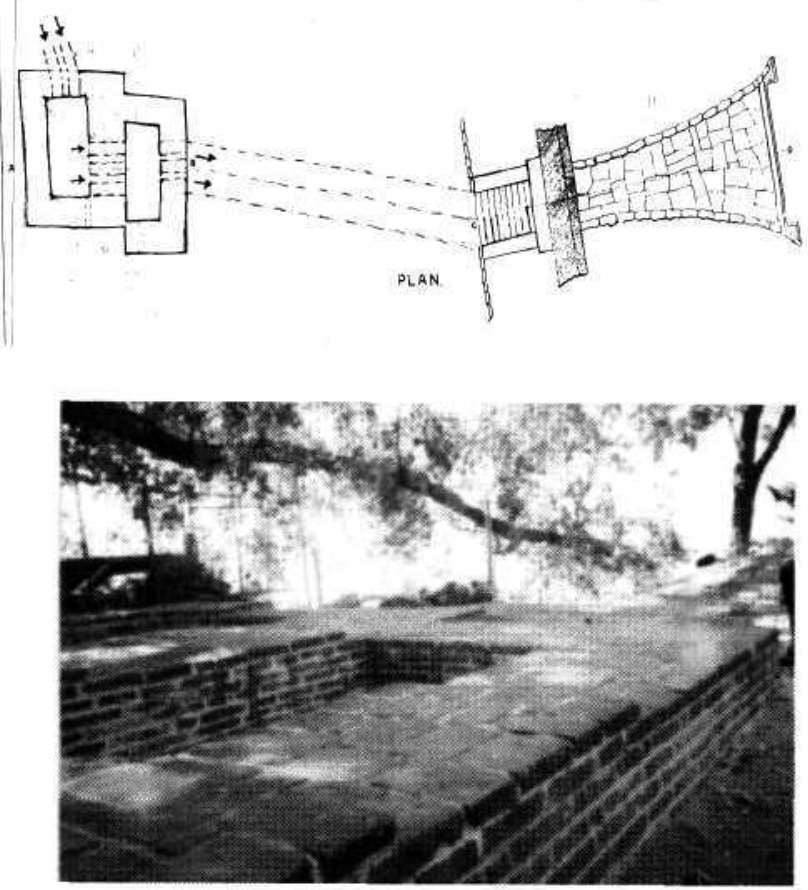

Figure. 6: Water diversion Bisokotuwa at Parakrama Samudra 


\section{Analyzing Existing Bisokotuwa as a Flow Regulator and A Meter using Modern Hydraulics}

Applying Bemouli's equation to Bisokotuwa (Figure 7)

From A to B

$H-h=f_{1}\left(Q, a_{1}\right)$

From B to C

$h=f_{2}\left(Q, a_{2}\right)$

Where

$\mathrm{H}=$ Height of water in vewa

h = Height of water in Bisokotuwa

Q = Water flow rate

$\mathrm{a}_{1}=$ Opening area of gate $\mathrm{G}_{1}$

$a_{2} \quad=$ Opening area of gate $G_{2}$

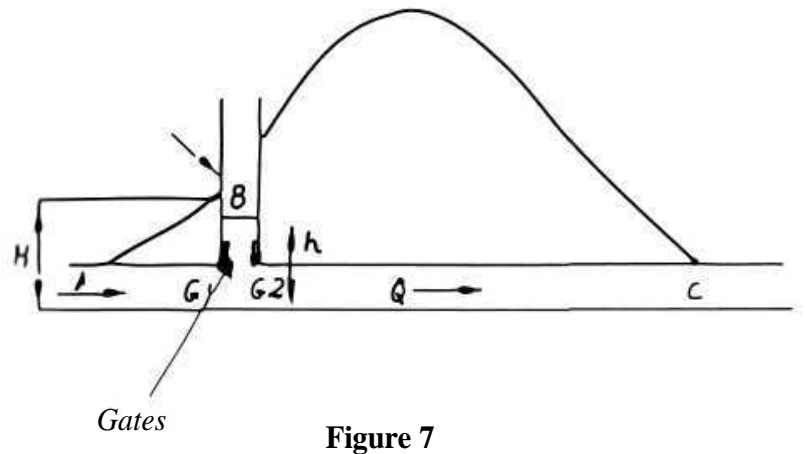

From equation (2) it is clear that for a particular set of values of $a_{2}$ and $h, Q$ will have a fixed value. Now, Bisokotuwa can be calibrated by finding the $Q$ values for various sets of $a_{2}$ and $h$ values i.e determining the two variable functions " $\mathrm{f}_{2}$ ", now if we want to have some particular $Q$ value then find the two values $a_{2}$ and $h$ accordingly and open the gate " $\mathrm{G}_{2}$ " now open the gate " $G$ " such that water level inside Bisokotuwa will be equal to the above " $h$ " value. Then the required water flow "Q" will take place, when the water level in the vewa goes down "h" value will also go down, then by increasing the opening area of gate "G1" "h" could be brought to the initial value i.e the water flow rate from the vewa could be kept constant independent of the height of water level in the vewa (for a particular range). Also once the bisokotuwa is calibrated as said above it could be used to measure the flow rate i.e when "h" and " $\mathrm{a}_{2}$ " known "Q" can be determined by calibration charts or graphs.

\section{Hydraulic Modelling to Estimate Flow Rate of Bisokotuwa (For New Designs)}

In this case the bisokotuwa with a particular gate opening, is considered with sluice and the entrance part ( part $\mathrm{ABC}$ in figure 8)

Now if a scale ( geometrically) model is made it is reasonable to assume that the flow rate $(\mathrm{Q})$ from "A " is a function of representative dimension (D) of the part ABC, water head in vewa $(H)$, density of water $(C)$, viscosity of water $(\mu)$ and acceleration due to gravity $(\mathrm{g})$ Then by using dimensional analysis it could be shown that

$\mathrm{H}^{-5 / 2} \mathrm{~g}^{-1 / 2} \mathrm{Q}=\mathrm{f}\left(\mathrm{H}^{-3 / 2}{ }^{-1} \mathrm{eg}^{-1 / 2} \mu, \mathrm{H}^{-1} \mathrm{D}\right)$

By manipulating " $e$ " it is possible to make a model which will have the same values for $\mathrm{H}^{-3 / 2}$ $e^{-1} \mathrm{~g}^{-1 / 2} \mathrm{u} \& \mathrm{H}^{-1} \mathrm{D}$ as in the proto type. Therefore flow rate could be predicted. Then the head loss from bisokotuwa also could be predicted.

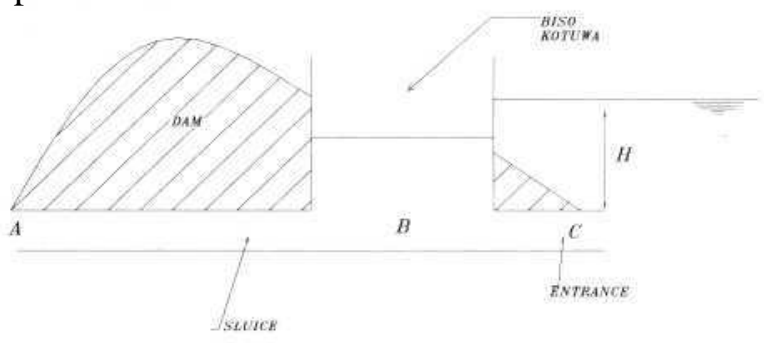

Figure 8: Schematic diagram of Bisokotuwa (relevant for hydraulic modeling )

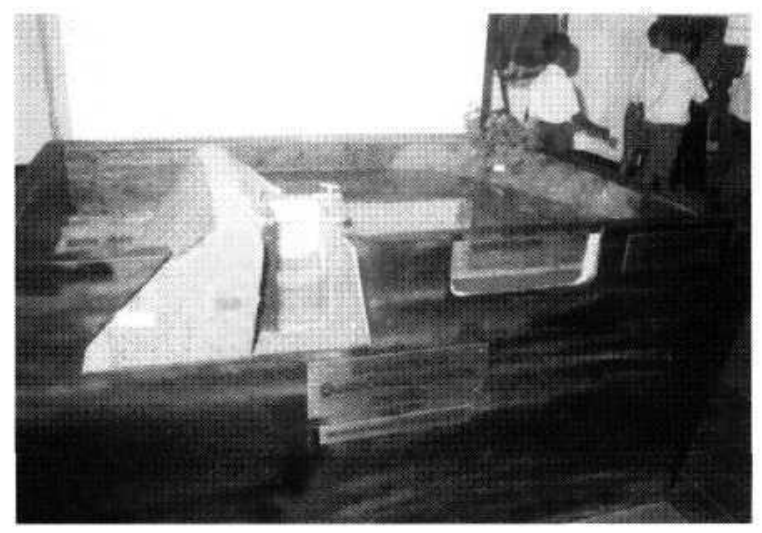

Figure 9: Working model of Bisokotuwa at National Museum 


\section{Conclusions}

The above analysis of the function of the Bisokotuwa can be used to emphasize the importance of this ancient hydraulic structure. Basically it serves as a surge tank, water pressure and velocity reducer and a gateretaining pit. Further this will lead us to investigate the possibility of using the Bisokotuwa as a flow meter and also to investigate further regarding ancient gates of the Bisokotuwa, which is not yet finalized. Also it should be mentioned here that we cannot exactly say that there was a gate for biso kotuwa. The above said main function of bisokotuwa could be achieved even without gates. Problem of gate should be analyzed by considering whole system i.e. the whole soilwater ecosystem.

\section{References}

(1) Ausadhahamy U. B "Vewa" - 1999 - P 36 - Sri Printers

(2) Jayawardena,s Bisokotuwa - A Note, 1980

(3) Mendis D. L. O Water Heritage of Sri Lanka, 2003 - Sri Lanka Pugwash Group, P 14

(4) Paker H Ancient Ceylon - 1984, P- 380 - Asian Educational Services.

(5) Fox \& Mc Donald - Introduction to fluid Mechanics ( Fifth edition) -2004 Wiley student edition. 